'Departamento de Psiquiatría, Escuela de Medicina, Universidad de Valparaíso. Valparaíso, Chile. ${ }^{2}$ Hospital del Salvador, Valparaíso. Valparaíso, Chile.

Trabajo no recibió financiamiento. El autor declara no tener conflictos de interés.

Recibido el 24 de junio de 2019, aceptado el 17 de diciembre de 2019.

Correspondencia a: Dr. Álvaro Cavieres 9 norte 915 , Viña del Mar. cavieres.alvaro@gmail.com

\section{El vínculo inflamatorio entre la enfermedad coronaria y la depresión}

\author{
ÁLVARO CAVIERES F. ${ }^{1,2}$
}

\section{Inflammation as a common pathogenic factor in coronary artery disease and depression}

A narrative review of the evidence regarding the role of inflammation in the pathophysiology of coronary heart disease and depression is presented. As a common pathogenic factor, inflammation could explain the frequent association of both diseases. We discuss the benefit of exercise and diet modifications in both conditions and the effectiveness and safety of selective serotonin reuptake inhibitors. Finally, we recommend screening for depressive symptoms in coronary patients and the search of cardiovascular risk factors in people with depression.

(Rev Med Chile 2020; 148: 224-232)

Key words: Coronary Disease; Depression; Inflammation.

\section{L} a cardiopatía coronaria y la depresión son dos patologías de alta prevalencia en nuestro medio, cuya frecuente presentación conjunta, empeora significativamente, el pronóstico de los pacientes. Es habitual atribuir esta asociación, a factores de riesgo comunes o a la consecuencia de presentar una enfermedad severa, actuando una condición como predisponente de la otra. En la actualidad, se ha propuesto que la inflamación, importante componente en la fisiopatología de ambos trastornos, podría ser un factor etiopatogénico inespecífico común, actuando como un nexo patológico entre el cerebro y el resto del organismo. La siguiente revisión bibliográfica descriptiva, no sistemática, presenta las principales evidencias respecto al rol de la inflamación en el origen de ambas enfermedades junto con sus implicancias terapéuticas y preventivas. Se privilegia los artículos de metaanálisis y capítulos de libros pertinentes, junto con publicaciones más recientes, intentando incluir también datos controversiales.

\section{Asociación depresión-cardiopatía isquémica}

La asociación entre depresión y enfermedad coronaria ha sido extensamente documentada ${ }^{1-4}$.
Un metaanálisis, con más de 10 mil casos de personas con el antecedente de un infarto al miocardio reciente, encontró que $20 \%$ presentaba también el diagnóstico de depresión ${ }^{5}$, una cifra similar a la reportada en pacientes con enfermedad coronaria crónica ${ }^{6}$. De manera inversa, al menos seis meta análisis, muestran una asociación entre depresión e incidencia futura de enfermedad cardiovascu$\operatorname{lar}^{7-12}$. En nuestro medio, un estudio realizado en el norte de Chile, encontró una prevalencia de depresión en los seis meses previos a un episodio coronario, cercana a $30 \%{ }^{13}$.

La frecuencia de la asociación descrita podría ser aún mayor si se consideran los casos depresivos leves o las etapas iniciales de la arterioesclerosis. Es necesario entender este proceso de manera bidireccional, donde la presencia de una condición, aumenta el riesgo de la otra, pero también como el resultado de factores biológicos comunes a ambas patologías, entre los cuales, la desregulación del proceso inflamatorio tendría un rol fisiopatológico central.

Al igual que la depresión y la enfermedad coronaria, la inflamación puede ser vista como un proceso continuo, cuyas características de intensidad, extensión y duración están determinadas por la interacción de múltiples variables. 
A diferencia de las características clásicas de la inflamación (enrojecimiento, aumento de volumen, calor y dolor), la neuroinflamación se refiere a una respuesta crónica de baja intensidad en la que células inmunes del cerebro (microglía, astrocitos y oligodendroglia) liberan mediadores inflamatorios sin invasión celular del parénquima ni provocar daño tisular ${ }^{14}$.

Los cambios inflamatorios establecen una comunicación bidireccional entre el cerebro y el resto del organismo. Las citokinas pueden atravesar la barrera hematoencefálica unidas a receptores en las células endoteliales de los vasos sanguíneos o en fibras nerviosas de aferentes del nervio vago. Una vez en el encéfalo, la activación de la microglía facilita el ingreso de leucocitos al cerebro, propagando el proceso inflamatorio ${ }^{15,16}$. Entre otras alteraciones, las citokinas proinflamatorias interfieren con el mecanismo inhibitorio del eje hipotálamo-hipófisis, manteniendo niveles elevados de cortisol en la periferia ${ }^{17,18}$.

\section{Factores de riesgo pro-inflamatorios}

El trastorno depresivo y la enfermedad coronaria, comparten un gran número de factores de riesgo. Sin pretender un listado exhaustivo, estos incluyen el sedentarismo, hábito tabáquico, obesidad, comorbilidades como la diabetes e hipertensión y estresores psicosociales ${ }^{19,20}$. A nivel fisiopatológico, todas estas variables son capaces de desencadenar mecanismos relacionados con un aumento de la actividad inflamatoria ${ }^{19,21}$.

El humo del cigarrillo contiene una gran cantidad de sustancias, las que al ser inhaladas, son capaces de generar una reacción inflamatoria en diversos tejidos, constituyendo un factor de riesgo de enfermedades crónicas, incluyendo las cardiovasculares ${ }^{22}$. Por otra parte, el tabaquismo es más frecuente entre las personas con diagnóstico de depresión que en la población general $^{23}$, aún cuando no se ha establecido si esta relación es uni o bidireccional. A su vez, la combinación tabaquismo-depresión se asocia a niveles circulantes de PCR, IL6 y TNF- $\alpha$ más elevados en comparación con fumadores sin depresión ${ }^{24}$.

Bajos niveles de ingesta de ácidos grasos poli insaturados, los que ejercen un efecto antiinflamatorio y protector del endotelio vascular, han sido asociados a una mayor prevalencia de patología cardíaca y depresión ${ }^{19}$. Por su parte, a la inversa de lo que ocurre con las frutas, vegetales, pescados y granos, la preferencia por alimentos ricos en azúcares refinados y carnes rojas se relacionan con mayores niveles circulantes de mediadores inflamatorios ${ }^{25,26}$.

Además de la composición nutricional, otra forma en que el aparato digestivo puede participar en la génesis de estados inflamatorios sistémicos es a través de la interacción entre la microbiota intestinal y el aparato inmune. En condiciones habituales, la flora bacteriana residente no es capaz de atravesar las paredes del intestino, pero se ha propuesto que en situaciones de permeabilidad aumentada ("leaky gut") ${ }^{27}$, podría llegar al sistema linfático y a la circulación general, desencadenando una respuesta inflamatoria ${ }^{28}$. Hay reportes de aumento de inmunoglobulinas plasmáticas anti bacterias comensales gramm (-) en personas con depresión ${ }^{29}$, mientras que la alteración de la composición de la microbiota intestinal ha sido relacionada con el aumento en la actividad inflamatoria y del riesgo de patología coronaria ${ }^{30}$. Si bien esta hipótesis ha recibido mucha atención, aún se requiere mayor evidencia para su confirmación $^{14,28}$.

Las personas sometidas a estresores psicosociales, ya sean agudos o crónicos, clínicamente significativos, muestran alteraciones inmunes como la elevación de los niveles circulantes de sustancias pro inflamatorias y disminución de las anti inflamatorias ${ }^{31-33}$. Por su parte, quienes han sido sometidos a traumas tempranos, en especial abusos y falta de cuidado, tienen mayor probabilidad de presentar estados de hipercortisolemia y elevación de niveles circulantes de citokinas pro inflamatorias ${ }^{34}$. La severidad de estas alteraciones está importantemente mediadas por factores genéticos ${ }^{33}$.

Normalmente, los glucocorticoides revierten los efectos de la inflamación, pero en situaciones de stress crónico, la desensibilización de los receptores de glucocorticoides, incluyendo a los ubicados en células inmunes ${ }^{35}$, impide este mecanismo regulatorio, aumentando la inflamación. Algo similar ocurre en el hipocampo ${ }^{36}$, con la pérdida del feedback negativo sobre la liberación de cortisol, conduciendo a estados de hipercortisolemia e hiperactividad del sistema nervioso autónomo. 


\section{Depresión e inflamación}

En el caso de la depresión, un número importante de factores podrían explicar el aumento de la actividad inflamatoria, la mayoría relacionados a estilos de vida poco saludables y presentes también en la patología cardíaca, como sedentarismo, tabaquismo, obesidad, alteraciones del sueño y nutricionales. Sin embargo, los factores más importantes son los relativos al stress y al trauma psicológico temprano ${ }^{37,38}$. Existe evidencia de que los estresores psicosociales pueden estimular la respuesta proinflamatoria, incluyendo aumentos en IL- 6 yTNF- $\alpha$, y de que estos cambios se asocian con los niveles de ansiedad experimentados por el sujeto ${ }^{31}$. Adicionalmente, el estrés también se acompaña de niveles reducidos de compuestos antiinflamatorios endógenos ${ }^{32}$.

Muchas personas con depresión exhiben aumentos en los niveles plasmáticos y urinarios de cortisol, junto con una disminución de la respuesta a la administración de dexametasona ${ }^{39}$. Estos cambios se han relacionado con la disminución del tamaño del hipocampo, y con mayor severidad y riesgo de recurrencia de la depresión ${ }^{40}$. A nivel cardíaco, estos mismos factores, aumentan el riesgo de mortalidad ${ }^{41,42}$. Es importante destacar que la disregulación del eje hipotálamo-hipofiso-adrenal, está vinculada más específicamente con estresores tempranos en la vida de las personas que con un diagnóstico específico ${ }^{43}$, y puede estar también presente en otros cuadros psiquiátricos, especialmente ansiosos ${ }^{44}$.

Un número importante de autores ha reportado la presencia de una actividad inflamatoria crónica y de bajo grado, en personas que cursan con un trastorno depresivo, caracterizada fundamental, pero no exclusivamente, por un aumento en las concentraciones plasmáticas de TNF- $\alpha$, interleukinas 1 y 6 y PCR ${ }^{15,45,46}$, especialmente empleando el método ultrasensible, que permite la detección de concentraciones más pequeñas. Ya sea por aferentes del nervio vago o penetrando a través de la barrera hematoencefálica ${ }^{16}$, estas moléculas proinflamatorias provocan la activación de la microglía, lo que a su vez, produce un aumento en la síntesis y liberación de citokinas ${ }^{15}$.

En el cerebro, las citokinas inflamatorias, pueden estimular la enzima indolamina 23 -dioxigenasa (IDO), disminuyendo la síntesis cerebral de serotonina, neurotransmisor clave en la regulación del estado de ánimo y aumentando la conversión del triptófano en kinurenina, con efecto neurotóxico y de ácido quinolínico con efecto glutaminérgico en los receptores $\mathrm{NMDA}^{14,47}$. Sin embargo, este mecanismo no está confirmado en todos los casos de depresión ${ }^{48,49}$.

El aumento de la actividad glutamatérgica, por efecto de las citokinas sobre la enzima IDO, o por una mayor liberación desde los astrocitos ${ }^{50}$, afecta el funcionamiento de los ganglios basales ${ }^{16}$, induciendo un conjunto de comportamientos, conocidos como "sickness behavior" 51 , los que incluyen la disminución de la actividad psicomotora y de la ingesta de agua y alimentos, sensación de fatiga y letargo y alteraciones cognitivas y del sueño. Estas manifestaciones se aproximan al fenotipo conductual de la depresión ${ }^{52}$.

Otra regiones afectadas son la amígdala, ínsula y cíngulo ${ }^{53}$, parte de un sistema de alarma, que detecta y responde a estímulos ambientales amenazantes, especialmente en el ámbito social ${ }^{54}$. El estado inflamatorio aumenta la sensibilidad a las señales del medio y amplifica la respuesta autonómica, provocando la sensación subjetiva de hipervigilancia y ansiedad en situaciones de potencial rechazo social ${ }^{16}$.

Es necesario considerar que las alteraciones inflamatorias no están presentes con igual intensidad en todas las personas que sufren depresión ${ }^{55} \mathrm{e}$ incluso podrían corresponder más específicamente, a los cuadros de tipo melancólico ${ }^{56}$. Otros autores han encontrado una correlación entre mayores niveles de citokinas proinflamatorias y mayor severidad de la depresión ${ }^{57,58}$. Igualmente, se ha reportado una relación entre mayor suicidalidad y mayor actividad inflamatoria ${ }^{59,60}$.

\section{Cardiopatía Isquémica e Inflamación}

Los procesos inflamatorios son centrales en el origen y progresión de la cardiopatía isquémica La interacción entre anormalidades inflamatorias y metabólicas puede provocar un daño al endotelio vascular, y junto a una hipercoagulabilidad, determinar la aparición de la ateroesclerosis ${ }^{61}$. La liberación del TNF- $\alpha$ desde el tejido adiposo, inicia una respuesta inflamatoria que puede extenderse luego a la vasculatura, y terminar afectando al miocardio ${ }^{62}$. Este estado inflamatorio crónico de baja intensidad, se relaciona 
también con la resistencia a la insulina, componente clave del Síndrome Metabólico, y una de las principales causas de morbimortalidad de origen cardiovascular.

El TNF- $\alpha$ es producido por los macrofagos en respuesta a diversos estímulos pro-inflamatorios como la obesidad, diabetes, hipertensión arterial e isquemia miocárdica. En niveles bajos puede ejercer efectos cardioprotectores, mientras que a concentraciones más elevadas tiene efectos deletéreos para el corazón. Se piensa que esto se debe a su capacidad de regular la entrada y las concentraciones intracelulares de calcio en el miocito ${ }^{63}$. Asimismo, se ha descrito que la progresión de la ateroesclerosis asociada a los estados inflamatorios crónicos puede ser atenuada con agentes anti TNF- $\alpha^{64}$.

La formación de ateromas, es una respuesta inflamatoria provocada por un exceso de lipoproteínas de baja densidad (LDL) plasmáticas que penetran las paredes vasculares y son oxidadas en el espacio subendotelial. En condiciones desfavorables, inducidas por el tabaquismo, hipertensión, hiperglicemia o dislipidemia, entre otros, se desencadena una situación de stress oxidativo que supera las capacidades adaptativas del organismo. El exceso de LDL oxidado es removido por macrófagos activados, los que junto a otras células inmunes, desencadenan una respuesta inflamatoria que contribuye al engrosamiento del ateroma, daño endotelial y ruptura final de la placa ateromatosa ${ }^{65}$.

Las citokinas, junto a otras sustancias proinflamatorias, también tienen un rol preponderante en la fisiopatología de la enfermedad isquémica. Uno de los mecanismos involucrados, es la activación de NF- $\kappa \mathrm{B}$, un factor de transcripción, cuya forma inactiva, formada por varias subunidades, se encuentra en el citoplasma de casi todas las células del organismo. Los mediadores inflamatorios catalizan la proteólisis parcial de la macromolécula, permitiendo su ingreso al núcleo, donde promueve la síntesis de proteínas involucradas tanto en la cardioprotección como en la patología cardíaca. De esta forma, NF- $\kappa B$ juega un rol modulatorio del compromiso de la integridad funcional del miocardio, en presencia de los cambios inflamatorios relacionados con la enfermedad isquémica ${ }^{66}$.

Diversas publicaciones han reportado alteraciones en la activación y agregación plaquetaria y en la cascada de coagulación en personas con diagnóstico de depresión ${ }^{21}$. Estas alteraciones podrían contribuir a una mayor formación de ateromas y coagulación de la sangre y por lo tanto, a un aumento en el riesgo de infartos. Sin embargo, no todos los estudios confirman estos hallazgos ${ }^{67}$, lo que lleva a la necesidad de diferenciar entre distintas formas de depresión y a identificar la presencia de otros factores mediadores.

\section{Manejo de la depresión en el paciente coronario}

La presencia de un episodio depresivo, no solo aumenta el riesgo de padecer un evento coronario $^{68}$, sino que además empeora el pronóstico del mismo, aún controlando otras variables ${ }^{69-71}$. Atendiendo a lo anterior, la detección de síntomas depresivos, por medio de una entrevista clínica, o al menos, la aplicación de una encuesta de tamizaje $^{72,73}$, debería ser aplicado a todas las personas con una enfermedad coronaria ${ }^{74}$.

El manejo de todos los episodios depresivos debiera siempre considerar los aspectos psicológicos y sociales del paciente, junto con la instalación de un tratamiento farmacológico adecuado. Las intervenciones psicosociales comprenden una amplia gama de técnicas y objetivos que van desde lo general, como educación en temas de salud, modificación de estilos de vida, afrontamiento de situaciones estresantes, ayuda en la resolución de problemas, hasta las psicoterapias propiamente tales. Revisiones sistemáticas del tema concluyen que estas estrategias disminuyen de manera pequeña a moderada los síntomas depresivos, pero sin mostrar beneficios en la morbilidad cardíaca ${ }^{75,76}$.

Mientras que no cabe duda de los beneficios de las dietas estilo Mediterráneo o del aumento en general, de la ingesta de frutas, verduras y fibras, en la disminución de los niveles circulantes de mediadores inflamatorios y del riesgo cardiovascular ${ }^{77,78}$, la evidencia respecto del efecto de estas medidas en el tratamiento del trastorno depresivo, si bien favorable, requiere de mayor confirmación ${ }^{79-81}$, al igual que la suplementación con ácidos grasos poliinsaturados ${ }^{82}$.

Respecto del ejercicio, existen reportes de una relación inversa entre el grado de actividad física y la presencia de síntomas depresivos en la población general, con alguna evidencia de un mayor 
riego de depresión futura asociada al sedentarismo. El ejercicio físico agudo intenso desencadena una respuesta inflamatoria y un posterior efecto antiinflamatorio, mientras que la actividad física regular disminuye la inflamación sistémica a través de este mecanismo homostático ${ }^{83,84}$. El beneficio del aumento del ejercicio para las personas con diagnóstico de depresión ha sido considerado desde poco a muy significativo, dependiendo de la metodología empleada. En particular, estudios con personas con depresión y enfermedad cardiovascular muestran resultados favorables para ambas condiciones.

Los fármacos antidepresivos difieren respecto de sus mecanismos de acción y perfil de efectos adversos, pero presentan efectividades comparables, al menos para los casos leves y moderados, existiendo reportes que asocian su administración con la disminución de los niveles circulantes de citokinas pro inflamatorias, independiente del efecto antidepresivo ${ }^{85}$. Sin embargo, en el caso de los pacientes coronarios se recomienda evitar el uso de fármacos tricíclicos por su riesgo de hipotensión ortóstatica y prolongación del QT, y de antidepresivos duales, asociados a hipertensión. Tampoco están recomendados los antiinflamatorios no-estoridales, con posible efecto antidepresivo, pero inseguros desde el punto de vista cardiovascular $^{86}$, mientras que las terapias con inhibidores de citokinas requieren de confirmación de sus beneficios psiquiátricos y cardiológicos ${ }^{64}$.

Existe evidencia de que los inhibidores selectivos de la recaptura de serotonina (ISRS),además de su seguridad y efectividad, mejoran la función endotelial, disminuyen los niveles de marcadores de inflamación ${ }^{87}$ y se asocian a menores tasas de rehospitalización ${ }^{88}$ en personas con depresión y patología coronaria. Específicamente, sertralina, destaca por su facilidad de uso, buen efecto terapéutico, bajo riesgo de interacciones farmacológicas, además de una posible utilidad como antiagregante plaquetario. El estudio SADHART, realizado en pacientes hospitalizados por un infarto miocárdico o angina inestable, demostró la seguridad de su uso, y efecto favorable, especialmente en cuadros depresivos más severos ${ }^{89}$.

Una de las investigaciones con mejor diseño metodológico, incluyó a personas con depresión y/o bajo apoyo psicosocial que hubieran sufrido recientemente un infarto al miocardio. Los participantes fueron asignados aleatoriamente al tratamiento habitual o a una intervención psicoterapéutica. Adicionalmente, los casos de depresión más severos en ambos grupos fueron tratados con antidepresivos. Si bien ambos grupos mostraron la misma tasa de re-infarto a los 29 meses $^{90}$, el outcome principal del estudio, hubo diferencias favorables para las personas que recibieron antidepresivos $^{91}$. Asimismo, quienes no mejoraron de sus síntomas depresivos presentaron una mayor mortalidad que el resto de los pacientes ${ }^{92}$. Estos resultados deben, sin embargo, interpretarse con cautela, ya que no corresponden al objetivo inicial del estudio.

\section{Comentarios}

El trastorno depresivo mayor y la patología coronaria son ampliamente prevalentes en nuestro medio y causantes de graves consecuencias biológicas, sociales y económicas. Las alteraciones de la respuesta inflamatoria descritas se asocian a su alta incidencia y comorbilidad, por lo que se hace necesaria su mejor comprensión.

Desde un punto de vista preventivo, es indudable el beneficio de adoptar estilos de vida más saludables, eliminando factores de riesgo de cardiopatía coronaria. En el caso de la depresión, la utilidad de estas recomendaciones, si bien igualmente saludables, no está claramente establecida.

Es deseable el monitoreo de síntomas depresivos en toda persona con síntomas coronarios. De igual manera, en los pacientes con depresión la evaluación psiquiátrica debiera incluir preguntas acerca de factores de riesgo cardiovascular y la solicitud de exámenes bioquímicos básicos, considerando eventualmente incorporar la determinación de PCR ultrasensible, como indicador del estado inflamatorio sistémico.

En la actualidad no existen marcadores periféricos que reflejen confiablemente la presencia de alteraciones inflamatorias en el cerebro, ni sus posibles consecuencias. A la espera de resultados de estudios translacionales y otros orientados a la clínica, que orienten de mejor manera una terapéutica individualizada, la recomendación vigente para el tratamiento de los casos de depresión asociada a patología coronaria es el manejo integral con modificación de estilos de vida poco saludables, apoyo psicológico y uso de antidepresivos inhibidores de la recaptura de serotonina. 


\section{Referencias}

1. Rudisch B, Nemeroff CB. Epidemiology of comorbid coronary artery disease and depression. Biol Psychiatry 2003; 54 (3): 227-40.

2. Chauvet-Gélinier J-C, Trojak B, Vergès-Patois B, Cottin Y, Bonin B. Review on depression and coronary heart disease. Arch Cardiovasc Dis 2013; 106 (2): 103-10.

3. Jørgensen TSH, Mårtensson S, Ibfelt EH, Jørgensen MB, Wium-Andersen IK, Wium-Andersen MK, et al. Time trend in depression diagnoses among acute coronary syndrome patients and a reference population from 2001 to 2009 in Denmark. Nord J Psychiatry 2016; 70 (5): 335-41.

4. Carney RM, Freedland KE. Depression and coronary heart disease. Nat Rev Cardiol 2017; 14 (3): 145-55.

5. Thombs BD, Bass EB, Ford DE, Stewart KJ, Tsilidis KK, Patel U, et al. Prevalence of depression in survivors of acute myocardial infarction. J Gen Intern Med 2006; 21 (1): 30-8.

6. Carney RM, Freedland KE. Depression in patients with coronary heart disease. Am J Med 2008; 121 (11 Suppl 2): S20-7.

7. Gan Y, Gong Y, Tong X, Sun H, Cong Y, Dong X, et al. Depression and the risk of coronary heart disease: a meta-analysis of prospective cohort studies. BMC Psychiatry 2014; 14 (1): 371.

8. Wulsin LR, Singal BM. Do depressive symptoms increase the risk for the onset of coronary disease? A systematic quantitative review. Psychosom Med 2003; 65 (2): 201-10.

9. Rugulies R. Depression as a predictor for coronary heart disease. a review and meta-analysis. Am J Prev Med 2002; 23 (1): 51-61.

10. Van der Kooy K, van Hout H, Marwijk H, Marten H, Stehouwer C, Beekman A. Depression and the risk for cardiovascular diseases: systematic review and meta analysis. Int J Geriatr Psychiatry 2007; 22 (7): 613-26.

11. Nicholson A, Kuper H, Hemingway H. Depression as an aetiologic and prognostic factor in coronary heart disease: a meta-analysis of 6362 events among 146538 participants in 54 observational studies. Eur Heart J 2006; 27 (23): 2763-74.

12. Cuijpers P, Smit F. Excess mortality in depression: a meta-analysis of community studies. J Affect Disord 2002; 72 (3): 227-36.

13. Araya A MV, Madariaga A C, Ureta V C, Tomé B M, Bustos M C. La depresión mayor como nuevo factor de riesgo en la cardiopatía coronaria en Chile. Rev Med Chile 2002; 130 (11): 1249-56.

14. Leonard BE. Inflammation and depression: a causal or coincidental link to the pathophysiology? Acta Neuropsychiatr 2018; 30 (1): 1-16.

15. D’Mello C, Swain MG. Immune-to-Brain Communication Pathways in Inflammation-Associated Sickness and Depression. Curr Top Behav Neurosci 2017 2017; 31: 73-94.

16. Miller AH, Haroon E, Raison CL, Felger JC. Cytokine targets in the brain: impact on neurotransmitters and neurocircuits. Depress Anxiety 2013; 30 (4): 297-306.

17. Raison CL, Miller AH. When not enough is too much: the role of insufficient glucocorticoid signaling in the pathophysiology of stress-related disorders. Am J Psychiatry 2003; 160 (9): 1554-65.

18. Merkulov VM, Merkulova TI, Bondar NP. Mechanisms of brain glucocorticoid resistance in stress-induced psychopathologies. Biochem [Internet] 2017; 82 (3): 351-65.

19. Skala JA, Freedland KE, Carney RM. Coronary heart disease and depression: a review of recent mechanistic research. Can J Psychiatry 2006; 51(12): 738-45.

20. Lett HS, Blumenthal JA, Babyak MA, Sherwood A, Strauman T, Robins C, et al. Depression as a risk factor for coronary artery disease: evidence, mechanisms, and treatment. Psychosom Med 2004; 66 (3): 305-15.

21. Nemeroff CB, Goldschmidt-Clermont PJ. Heartache and heartbreak-the link between depression and cardiovascular disease. Nat Rev Cardiol 2012; 9 (9): 526-39.

22. Messner B, Bernhard D. Smoking and Cardiovascular Disease. Arterioscler Thromb Vasc Biol 2014; 34 (3): 509-15.

23. Lasser K, Boyd JW, Woolhandler S, Himmelstein DU, McCormick D, Bor DH. Smoking and mental illness: A population-based prevalence study. JAMA 2000; 284 (20): 2606-10.

24. Nunes SOV, Vargas HO, Brum J, Prado E, Vargas MM, de Castro MRP, et al. A comparison of inflammatory markers in depressed and nondepressed smokers. Nicotine Tob Res 2012; 14 (5): 540-6.

25. Whalen KA, McCullough ML, Flanders WD, Hartman TJ, Judd S, Bostick RM. Paleolithic and Mediterranean Diet Pattern Scores Are Inversely Associated with Biomarkers of Inflammation and Oxidative Balance in Adults. J Nutr 2016146 (6): 1217-26.

26. Lopez-Garcia E, Schulze MB, Fung TT, Meigs JB, Rifai $\mathrm{N}$, Manson JE, et al. Major dietary patterns are related to plasma concentrations of markers of inflammation and endothelial dysfunction. Am J Clin Nutr 2004; 80 (4): 1029-35.

27. Mu Q, Kirby J, Reilly CM, Luo XM. Leaky Gut As a Danger Signal for Autoimmune Diseases. Front Immunol 2017; 8: 598. 
28. Slyepchenko A, Maes M, Jacka FN, Köhler CA, Barichello T, McIntyre RS, et al. Gut Microbiota, Bacterial Translocation, and Interactions with Diet: Pathophysiological Links between Major Depressive Disorder and Non-Communicable Medical Comorbidities. Psychother Psychosom 2017; 86 (1): 31-46.

29. Maes M, Kubera M, Leunis JC, Berk M. Increased IgA and IgM responses against gut commensals in chronic depression: Further evidence for increased bacterial translocation or leaky gut. J Affect Disord 2012; 141 (1): 55-62.

30. Zununi Vahed S, Barzegari A, Zuluaga M, Letourneur D, Pavon-Djavid G. Myocardial infarction and gut microbiota: An incidental connection. Pharmacol Res 2018; 129: 308-17.

31. Maes M, Song C, Lin A, De Jongh R, Van Gastel A, Kenis $G$, et al. The effects of psychological stress on humans: increased production of pro-inflammatory cytokines and th1-like response in stress-induced anxiety. Cytokine 1998; 10 (4): 313-8.

32. Song C, Kenis G, van Gastel A, Bosmans E, Lin A, de Jong $\mathrm{R}$, et al. Influence of psychological stress on immune-inflammatory variables in normal humans. Part II. Altered serum concentrations of natural anti-inflammatory agents and soluble membrane antigens of monocytes and T lymphocytes. Psychiatry Res 1999; 85 (3): 293-303.

33. Slavich GM, O’Donovan A, Epel ES, Kemeny ME. Black sheep get the blues: a psychobiological model of social rejection and depression. Neurosci Biobehav Rev 2010; 35 (1): 39-45.

34. Danese A, Pariante CM, Caspi A, Taylor A, Poulton R. Childhood maltreatment predicts adult inflammation in a life-course study. Proc Natl Acad Sci USA 2007; 104 (4): 1319-24.

35. Quan N, Avitsur R, Stark JL, He L, Shah M, Caligiuri $\mathrm{M}$, et al. Social stress increases the susceptibility to endotoxic shock. J Neuroimmunol 2001; 115 (1-2): 36-45.

36. Quan N, He L, Lai W, Shen T, Herkenham M. Induction of IkappaBalpha mRNA expression in the brain by glucocorticoids: a negative feedback mechanism for immune-to-brain signaling. J Neurosci 2000; 20 (17): 6473-7.

37. Mandelli L, Petrelli C, Serretti A. The role of specific early trauma in adult depression: A meta-analysis of published literature. Childhood trauma and adult depression. Eur Psychiatry 2015; 30 (6): 665-80.

38. Nemeroff CB. Paradise Lost: The Neurobiological and Clinical Consequences of Child Abuse and Neglect. Neuron 2016; 89 (5): 892-909.

39. Nikkheslat N, Pariante CM, Zunszain PA. Neuroendo- crine Abnormalities in Major Depression: An Insight Into Glucocorticoids, Cytokines, and the Kynurenine Pathway. Inflamm Immun Depress 2018;45-60.

40. Sheline Y, Mittler B, Mintun M. The hippocampus and depression. Eur Psychiatry 2002; 17: 300-5.

41. Jokinen J, Nordström P. HPA axis hyperactivity and cardiovascular mortality in mood disorder inpatients. J Affect Disord 2009; 116 (1-2): 88-92.

42. Vogelzangs N, Beekman ATF, Milaneschi Y, Bandinelli S, Ferrucci L, Penninx BWJH. Urinary cortisol and sixyear risk of all-cause and cardiovascular mortality. J Clin Endocrinol Metab 2010; 95 (11): 4959-64.

43. Ventriglio A, Gentile A, Baldessarini RJ, Bellomo A. Early-life stress and psychiatric disorders: epidemiology, neurobiology and innovative pharmacological targets. Curr Pharm Des 2015; 21 (11): 1379-87.

44. Zorn JV, Schür RR, Boks MP, Kahn RS, Joëls M, Vinkers $\mathrm{CH}$. Cortisol stress reactivity across psychiatric disorders: A systematic review and meta-analysis. Psychoneuroendocrinology 2017; 77: 25-36.

45. Liu CS, Adibfar A, Herrmann N, Gallagher D, Lanctôt KL. Evidence for Inflammation-Associated Depression. In: Current topics in behavioral neurosciences 2016. p. 3-30.

46. Patel A. Review: the role of inflammation in depression. Psychiatr Danub 2013; 25 Suppl 2: S216-23.

47. M. Schmidt F, C. Kirkby K, Lichtblau N. Inflammation and Immune Regulation as Potential Drug Targets in Antidepressant Treatment. Curr Neuropharmacol 2016; 14 (7): 674-87.

48. Fukuda K. Etiological classification of depression based on the enzymes of tryptophan metabolism. BMC Psychiatry 2014; 14(1):372.

49. Quak J, Doornbos B, Roest AM, Duivis HE, Vogelzangs $\mathrm{N}$, Nolen WA, et al. Does tryptophan degradation along the kynurenine pathway mediate the association between pro-inflammatory immune activity and depressive symptoms? Psychoneuroendocrinology 2014; 45: 202-10.

50. Ida T, Hara M, Nakamura Y, Kozaki S, Tsunoda S, Ihara H. Cytokine-induced enhancement of calcium-dependent glutamate release from astrocytes mediated by nitric oxide. Neurosci Lett 2008; 432 (3): 232-6.

51. Dantzer R, O’Connor JC, Freund GG, Johnson RW, Kelley KW. From inflammation to sickness and depression: when the immune system subjugates the brain. Nat Rev Neurosci 2008; 9 (1): 46-56.

52. Dantzer R. Cytokine, Sickness Behavior, and Depression. Immunol Allergy Clin North Am 2009; 29 (2): 247.

53. Harrison NA, Brydon L, Walker C, Gray MA, Steptoe A, Critchley HD. Inflammation Causes Mood Changes 
Through Alterations in Subgenual Cingulate Activity and Mesolimbic Connectivity. Biol Psychiatry 2009; 66 (5): 407-14.

54. Eisenberger NI, Lieberman MD. Why rejection hurts: a common neural alarm system for physical and social pain. Trends Cogn Sci 2004; 8 (7): 294-300.

55. Steptoe A, Kunz-Ebrecht SR, Owen N. Lack of association between depressive symptoms and markers of immune and vascular inflammation in middle-aged men and women. Psychol Med 2003; 33 (4): 667-74.

56. Lotrich FE. Inflammatory cytokine-associated depression. Brain Res 2015; 1617: 113-25.

57. Maes M, Bosmans E, De Jongh R, Kenis G, Vandoolaeghe E, Neels H. Increased serum il-6 and il-1 receptor antagonist concentrations in major depression and treatment resistant depression. Cytokine 1997; 9 (11): 853-8.

58. Baune BT, Dannlowski U, Domschke K, Janssen DGA, Jordan MA, Ohrmann P, et al. The interleukin 1 beta (IL1B) gene is associated with failure to achieve remission and impaired emotion processing in major depression. Biol Psychiatry 2010; 67 (6): 543-9.

59. Serafini G, Pompili M, Elena Seretti M, Stefani H, Palermo M, Coryell W, et al. The role of inflammatory cytokines in suicidal behavior: A systematic review. Eur Neuropsychopharmacol 2013; 23 (12): 1672-86.

60. Black C, Miller BJ. Meta-Analysis of Cytokines and Chemokines in Suicidality: Distinguishing Suicidal Versus Nonsuicidal Patients. Biol Psychiatry 2015; 78 (1): 28-37.

61. Grandl G, Wolfrum C. Hemostasis, endothelial stress, inflammation, and the metabolic syndrome. Semin Immunopathol 2018; 40 (2): 215-24.

62. Kuroda M, Sakaue H. Adipocyte Death and Chronic Inflammation in Obesity. J Med Investig 2017; 64 (3.4): 193-6.

63. Bartekova M, Radosinska J, Jelemensky M, Dhalla NS. Role of cytokines and inflammation in heart function during health and disease. Heart Fail Rev 2018; 23 (5): 733-58.

64. Hartman MHT, Groot HE, Leach IM, Karper JC, van der Harst P. Translational overview of cytokine inhibition in acute myocardial infarction and chronic heart failure. Trends Cardiovasc Med 2018; 28 (6): 369-79.

65. Wu M-Y, Li C-J, Hou M-F, Chu P-Y. New Insights into the Role of Inflammation in the Pathogenesis of Atherosclerosis. Int J Mol Sci 2017; 18 (10).

66. Ellulu MS, Patimah I, Khaza'ai H, Rahmat A, Abed Y, Ali F. Atherosclerotic cardiovascular disease: a review of initiators and protective factors. Inflammopharmacology 2016; 24 (1): 1-10.
67. von Känel R. Platelet hyperactivity in clinical depression and the beneficial effect of antidepressant drug treatment: how strong is the evidence? Acta Psychiatr Scand 2004; 110 (3): 163-77.

68. Penninx BWJH, Beekman ATF, Honig A, Deeg DJH, Schoevers RA, van Eijk JTM, et al. Depression and Cardiac Mortality. Arch Gen Psychiatry 2001; 58 (3): 221.

69. Frasure-Smith N, Lespérance F, Juneau M, Talajic M, Bourassa MG. Gender, depression, and one-year prognosis after myocardial infarction. Psychosom Med 1999; 61 (1): 26-37.

70. Lichtman JH, Froelicher ES, Blumenthal JA, Carney RM, Doering LV, Frasure-Smith N, et al. Depression as a Risk Factor for Poor Prognosis Among Patients With Acute Coronary Syndrome: Systematic Review and Recommendations. Circulation 2014; 129 (12): 1350-69.

71. Bush DE, Ziegelstein RC, Tayback M, Richter D, Stevens $\mathrm{S}$, Zahalsky $\mathrm{H}$, et al. Even minimal symptoms of depression increase mortality risk after acute myocardial infarction. Am J Cardiol 2001; 88 (4): 337-41.

72. Kroenke K, Spitzer RL, Williams JBW. The Patient Health Questionnaire-2: validity of a two-item depression screener. Med Care 2003; 41 (11): 1284-92.

73. Kroenke K, Spitzer RL, Williams JB. The PHQ-9: validity of a brief depression severity measure. J Gen Intern Med 2001; 16 (9): 606-13.

74. Lichtman JH, Bigger JT, Blumenthal JA, Frasure-Smith N, Kaufmann PG, Lespérance F, et al. Depression and Coronary Heart Disease. Circulation 2008; 118(17):1768-75.

75. Richards SH, Anderson L, Jenkinson CE, Whalley B, Rees K, Davies P, et al. Psychological interventions for coronary heart disease: Cochrane systematic review and meta-analysis. Eur J Prev Cardiol 2018; 25 (3): 247-59.

76. Ski CF, Jelinek M, Jackson AC, Murphy BM, Thompson DR. Psychosocial interventions for patients with coronary heart disease and depression: A systematic review and meta-analysis. Eur J Cardiovasc Nurs 2016; 15 (5): 305-16.

77. Jenkins DJA, Kendall CWC, Marchie A, Faulkner DA, Wong JMW, de Souza R, et al. Effects of a Dietary Portfolio of Cholesterol-Lowering Foods vs Lovastatin on Serum Lipids and C-Reactive Protein. JAMA 2003; 290 (4): 502 .

78. Esposito K, Marfella R, Ciotola M, Di Palo C, Giugliano F, Giugliano G, et al. Effect of a mediterranean-style diet on endothelial dysfunction and markers of vascular inflammation in the metabolic syndrome: a randomized trial. JAMA 2004; 292 (12): 1440-6.

79. García-Toro M, Vicens-Pons E, Gili M, Roca M, Serra- 
no-Ripoll MJ, Vives M, et al. Obesity, metabolic syndrome and Mediterranean diet: Impact on depression outcome. J Affect Disord 2016; 194: 105-8.

80. Null G, Pennesi L. Diet and lifestyle intervention on chronic moderate to severe depression and anxiety and other chronic conditions. Complement Ther Clin Pract 2017; 29: 189-93.

81. Opie RS, O’Neil A, Itsiopoulos C, Jacka FN. The impact of whole-of-diet interventions on depression and anxiety: a systematic review of randomised controlled trials. Public Health Nutr 2015; 18 (11): 2074-93.

82. Zuzarte P, Duong A, Figueira ML, Costa-Vitali A, Scola G. Current Therapeutic Approaches for Targeting Inflammation in Depression and Cardiovascular Disease. Curr Drug Metab 2018; 19 (8): 674-87.

83. Walsh NP, Gleeson M, Shephard RJ, Gleeson M, Woods JA, Bishop NC, et al. Position statement. Part one: Immune function and exercise. Exerc Immunol Rev 2011; 17: 6-63.

84. Ostrowski K, Rohde T, Asp S, Schjerling P, Pedersen BK. Pro- and anti-inflammatory cytokine balance in strenuous exercise in humans. J Physiol 1999; 515 (1): 287-91.

85. Strawbridge R, Arnone D, Danese A, Papadopoulos A, Herane Vives A, Cleare AJ. Inflammation and clinical response to treatment in depression: A meta-analysis. Eur Neuropsychopharmacol 2015; 25 (10): 1532-43.

86. Varas-Lorenzo C, Riera-Guardia N, Calingaert B, Castellsague J, Salvo F, Nicotra F, et al. Myocardial infarction and individual nonsteroidal anti-inflammatory drugs meta-analysis of observational studies. Pharma- coepidemiol Drug Saf 2013; 22 (6): 559-70.

87. Pizzi C, Mancini S, Angeloni L, Fontana F, Manzoli L, Costa GM. Effects of Selective Serotonin Reuptake Inhibitor Therapy on Endothelial Function and Inflammatory Markers in Patients With Coronary Heart Disease. Clin Pharmacol Ther 2009;86(5):527-32.

88. Mazza M, Lotrionte M, Biondi-Zoccai G, Abbate A, Sheiban I, Romagnoli E. Selective serotonin reuptake inhibitors provide significant lower re-hospitalization rates in patients recovering from acute coronary syndromes: evidence from a meta-analysis. J Psychopharmacol 2010; 24 (12): 1785-92.

89. Glassman AH, O'Connor CM, Califf RM, Swedberg K, Schwartz P, Bigger JT, et al. Sertraline treatment of major depression in patients with acute MI or unstable angina. JAMA 2002; 288 (6): 701-9.

90. ENRICHD Investigators. Enhancing Recovery in Coronary Heart Disease (ENRICHD) study intervention: rationale and design. Psychosom Med 2001; 63 (5): 747-55.

91. Berkman LF, Blumenthal J, Burg M, Carney RM, Catellier D, Cowan MJ, et al. Effects of treating depression and low perceived social support on clinical events after myocardial infarction: the Enhancing Recovery in Coronary Heart Disease Patients (ENRICHD) Randomized Trial. JAMA 2003; 289 (23): 3106-16.

92. Carney RM, Blumenthal JA, Freedland KE, Youngblood M, Veith RC, Burg MM, et al. Depression and Late Mortality After Myocardial Infarction in the Enhancing Recovery in Coronary Heart Disease (ENRICHD) Study. Psychosom Med 2004; 66 (4): 466-74. 